\title{
Homebirth and homecare during COVID-19
}

\author{
Revekka Ziogou ${ }^{1}$, Katerina Zografou ${ }^{2}$
}

\section{Dear Editor,}

In the middle of the COVID-19 pandemic, even though a disease should be the number one reason to be admitted to a hospital, it turns out to be childbirth, in which midwives play an important role ${ }^{1,2}$. Within the COVID-19 pandemic, a pregnant woman who had planned to give birth at the hospital may have second thoughts and may get stressed about giving birth in such a hospital environment. Prenatal anxiety and depression are associated with preterm births, low birthweight babies, and small head circumference ${ }^{3}$.

Due to COVID-19, perinatal care has to be tailored to provide women with the best and most safe care. Midwives should offer alternatives to sustain and protect maternal and neonatal health. First, telecare is an excellent option during the perinatal period, which results in a reduction of face-to-face visits ${ }^{4}$ and minimizes the cost of care ${ }^{5}$. Second, blood pressure, weight and fetal heart rates can be remotely monitored ${ }^{5,6}$. Information and education can also be provided through telecare. Pregnant women are familiar with the use of smartphones and technology, and they have a positive attitude towards remote home-monitoring?

A planned homebirth with a registered midwife could be an excellent and safe alternative for low-risk pregnant women. It is very important for both pregnant woman and midwife to have a birth plan, instead of a last-minute decision, as the transfer to the nearest hospital has to be prearranged, in the rare case that something goes wrong. Fewer interventions, including episiotomy, epidural anaesthesia or delivery with a vacuum take place during a homebirth compared to planned hospital births ${ }^{8}$. The comfort of home, the right to choose and the continuity of care are some of the main reasons why mothers choose to give birth at home. Moreover, the benefit from their partner's presence and support are also very important ${ }^{9}$. During the postpartum period, a midwife may perform virtual or in-person home visits to check on mothers' and infants' health. Furthermore, home birth may cost less than a hospital birth ${ }^{10}$. Hospital and pharmaceutical expenses both for mother and infant are less when a woman delivers at home with a midwife.

Humanity has suffered many pandemics throughout its history, and this will probably not change in the future. As the Word Health Organization has declared 2020 the year of midwives, it is an excellent opportunity to establish a midwifery-led model of maternity care, which will minimize the cost and provide all women with high-quality perinatal care.

\section{REFERENGES}

1. Vermeulen J, Jokinen M. The European Midwives Association call for action to protect our midwives in delivering best care amidst the COVID-19 pandemic. European Journal of Midwifery. 2020;4(April). doi:10.18332/ejm/120443

2. Canadian Institute for Health Information. Hospital stays in Canada. https://www.cihi. $\mathrm{ca} / \mathrm{en} /$ hospital-stays-in-canada. Accessed April 20, 2020.

3. Grigoriadis S, Graves L, Peer M, et al. Maternal Anxiety During Pregnancy and the Association With Adverse Perinatal Outcomes: Systematic Review and Meta-Analysis. J Clin Psychiatry. 2018;79(5):17r12011. doi:10.4088/JCP.17r12011

4. Marko Kl, Ganju N, Krapf JM, et al. A Mobile Prenatal Care App to Reduce In-Person Visits: Prospective Controlled Trial JMIR Mhealth Uhealth. 2019;7(5):e10520. doi:10.2196/10520

5. Barbour DK, Nelson R, Esplin MS, Varner M, Clark ASE. A Randomised trial of prenatal care using telemedicine for low-risk pregnancies: patient-related cost and time savings. Am J Obstet Gynecol. 2017:S499. doi:10.1016/j.ajog.2016.11.782

\author{
AFFILIATION \\ 1 National Public Health \\ Organization-PHILOS, Refugee \\ Center, Alexandria, Greece \\ 2 Department of High-Risk \\ Pregnancies and Postpartum \\ Ward, General University Hospital \\ of Patras, Patra, Greece
}

\section{GORRESPONDENGE TO}

Revekka Ziogou. National Public Health Organization-PHILOS, Refugee Center, Alexandria, Greece. E-mail: ziogoureb@gmail.com

\section{KEYWORDS}

homebirth, telecare, midwife, perinatal care, COVID-19, virtual visit

Received: 21 April 2020 Accepted: 22 April 2020 
6. Pflugeisen BM, McCarren C, Poore S, Carlile M, Schroeder R. Virtual Visits: Managing prenatal care with modern technology MCN Am J Matern Child Nurs. 2016;41(1):24-30. doi:10.1097/NMC.0000000000000199

7. Pflugeisen BM, Mou J. Patient satisfaction with Virtual Obstetric Care Matern. Child Health J. 2017;21(7):1544-1551. doi:10.1007/s10995-017-2284-1

8. Sandall J, Soltani H, Gates S, Shennan A, Devane D. Midwife-led continuity models versus other models of care for childbearing women. Cochrane Database Syst Rev. 2016;4:CD004667. doi:10.1002/14651858.CD004667.pub5

9. Hauck Y, Nathan E, Ball C, Hutchinson M, Somerville S, Hornbuckle J, Doherty D. Women's reasons and perceptions around planning a homebirth with a registered midwife in Western Australia. Women Birth. 2020;33(1):e39-e47. doi:10.1016/j.wombi.2018.11.017

10. Janssen P, Mitton C, Aghajanian J. Costs of Planned Home vs. Hospital Birth in British Columbia Attended by Registered Midwives and Physicians. PLoS One. 2015;10(7):e0133524. doi:10.1371/journal.pone.0133524

\section{CONFLICTS OF INTEREST}

The authors have completed and submitted the ICMJE Form for Disclosure of Potential Conflicts of Interest and none was reported.

\section{FUNDING}

There was no source of funding for this research.

\section{PROVENANGE AND PEER REVIEW}

Not commissioned; internally peer reviewed. 